\section{Involved Field Radiotherapy}

Carol L. Armstrong

Child and Adolescent Psychiatry and Behavioral

Sciences, The Children's Hospital of

Philadelphia, Philadelphia, PA, USA

\section{Synonyms}

Clinical target volume; Conformal techniques; Partial field radiotherapy

\section{Definition}

Involved field radiotherapy delivers the full therapeutic dose of radiation from three dimensions to only the areas involved by the cancer, and usually a $1 \mathrm{~cm}$ or $2 \mathrm{~cm}$ surrounding the tumor or tumor bed post-resection. It is useful when the neoplasm is close to critical body tissues, organs, or structures because less normal tissue is in the field of radiation. It is used for several types of cancers, including lymphoma, esophageal, liver, pancreatic, lung, breast, prostate, and brain neoplasms.
Currently, delivery of radiation to the involved field can be done by several techniques, including conformal radiotherapy, intensity modulated radiotherapy (IMRT), and proton beam techniques such as proton pencil beam scanning. Total doses and dose fractions are typically higher than those given in whole brain treatments but lower than those used in radiosurgery. The clinical target volumes include the tumor volume and the presumed region of microscopic extension $(2 \mathrm{~cm}$ margin) (Kahn 1994). Doses are divided into fractions to decrease damage to healthy tissues, and fractions are divided into daily doses to allow DNA repair kinetics.

\section{Cross-References}

Late Effects of Radiation Therapy

\section{References and Readings}

Kahn, F. (1994). The physics of radiation therapy (2nd ed.). Baltimore: Williams \& Wilkins. 